\title{
EL USO DE LOS ESPACIOS PÚBLICOS EN LA CIUDAD DE HUANCAYO*
}

\author{
Arredondo Baquerizo, Freder Lorgio ${ }^{1}$ y Romero Sihuay, Carlos Antonio ${ }^{2}$
}

Facultad de Antropología de la Universidad Nacional del Centro del Perú

\begin{abstract}
RESUMEN
La investigación se centra en el uso de los espacios públicos en la ciudad de Huancayo, teniendo como objetivos específicos los siguientes: determinar los aspectos sociales y culturales que concurren en la utilización de los espacios públicos en la ciudad de Huancayo y diseñar una propuesta social para el uso adecuado de los espacios públicos en esta ciudad. La metodología utilizada es la hermenéutica, la descripción del problema, cómo el poblador de esta urbe utiliza estos espacios, especialmente algunos lugares escogidos por los investigadores; mercados, calles principales y días especiales del año. La interpretación de estos datos se ha realizado teniendo cuenta la teoría social y la percepción personal de los investigadores. Dentro de los resultados obtenidos, consideramos que existe un mal uso de los espacios públicos; debido a la existencia de una cultura de la trasgresión de las normas, y un goce clandestino por estos actos, del desorden y de la desorganización de parte de los pobladores y de las autoridades, además de un manejo del cuerpo socialmente construido que se ha adaptado a esta realidad. Se percibe la incipiente construcción de la ciudadanía, del irrespeto por el otro y el creciente individualismo frente al espíritu comunitario. El problema no es técnico, es social y cultural. En base a este diagnóstico, se puede buscar las soluciones.
\end{abstract}

Palabras clave: trasgresión, cultura criolla, manejo del cuerpo, construcción social, ciudadanía, responsabilidad social.

\section{THE USE OF THE PUBLIC SPACES IN HUANCAYO CITY}

\begin{abstract}
The searching is about the use of the public spaces in Huancayo city. To search the use of the public spaces in Huancayo city and design a social propose for the adequate use of the public spaces in Huancayo city. The methodology used is the hermeneutics, the description problem, how the resident of this town uses these spaces, especially some places chosed by the searchers; markets, main streets and special days of the year. The interpretations of these facts have beendone with the social theory and the personal perception of the searchers. from the results, we consider that exists a bad use of the public spaces, because the existence of cultural transgression, about the norms and a clandestine enjoyment by these acts, the disorder and the disorganization by the people and the authorities, besides a social body management, built that has adapted to this reality. It is perceived an incipient citizen construction, of disrespect by the other and the increase individualism against the collective spirit. The problem is not technical, it is social and cultural, in base of this diagnostic, we can look for the solutions.
\end{abstract}

Key words: transgression, criolla culture; body management, social built, citizenship, social responsibility

\footnotetext{
* Este trabajo de investigación fue recibido el 20/06/2008 retornado para su revisión 26/10/2009 y aprobado para su publicación 16/11/2009

${ }^{1}$ Email: arredondo.fl@gmail.com

2Email: kroma29@hotmail.com
} 


\section{INTRODUCCIÓN}

La ciudad de Huancayo es una urbe relativamente joven (en comparación a otras ciudades del Perú 0 de la Región) recién a partir del siglo XIX empieza a establecerse como una pequeña ciudad, es decir, Huancayo no tuvo un pasado colonial, sin embargo, a inicios del siglo XX, empieza su gran crecimiento, primero con la construcción del Ferrocarril Central (1908) y luego con la llegada de Centromín Perú en la década de los 20 del siglo pasado. Con la culminación de la Carretera Central en 1928; se convierte en un espacio en los andes peruanos que va a tener un gran desarrollo económico - comercial y social - demográfico por su articulación permanente y cercanía con la capital del país. Este hecho lo convierte en un gran foco de atracción para los pobladores de distintas partes de la región y de todo el país.

A mediados del siglo XX Huancayo se convierte en una ciudad intermedia entre otras regiones y ciudades como Huancavelica, Ayacucho, Satipo y la gran Lima, sirve como tránsito para la comercialización y migración, además de convertirse por su modernidad y por su especial configuración social en un lugar donde se puede migrar y lograr un desarrollo social y económico, en comparación a otros lugares más tradicionales y alejados que dificultan una movilidad social.

Debido a estas razones, la ciudad de Huancayo y otras provincias, distritos y pueblos de su entorno, comienzan a poblarse, debido al crecimiento demográfico y sobre todo al desplazamiento de familias y personas de distintas partes de la sierra, de la costa e incluso del oriente confluyen en este nuevo espacio. Pero, el crecimiento poblacional de Huancayo no va acompañada de una adecuada planificación urbana por parte de las distintas autoridades, muy por el contrario, en cierta medida como lo señala Matos Mar, para el caso de Lima, se va produciendo un desborde popular, en pequeña escala, la ciudad crece hacia los cuatro puntos cardinales, pero fundamentalmente hacia el norte (El Tambo) y hacia el sur (Chilca). La escasez de servicios básicos, de vivienda, de empleo; en la medida que la ciudad reafirma su característica de ser comercial y a través de este tipo de economía va logrando un relativo bienestar a su población, pero temas como el transporte automotor público y privado, la contaminación ambiental, la seguridad ciudadana, el manejo de residuos sólidos y líquidos, el comercio informal, el sistema de alcantarillado, el ordenamiento urbano, la construcción de áreas verdes, de recreación, el uso de los espacios públicos incluso el abastecimiento de agua potable y la construcción de una ciudadanía que se identifique con los problemas de su ciudad, son temas aún pendientes.

Respecto al transporte es notorio un gran desorden, existen 4 puentes y tres avenidas principales (Real, Huancavelica y Ferrocarril) que conectan la parte sur y norte de la ciudad, y ésta con otros distritos rurales que está atravesada por el río Shullcas. Diariamente en las horas punta, en el momento que las personas salen de sus centro de trabajo y los estudiantes acuden 0 salen de sus centros de estudios, se producen embotellamientos de vehículos en el centros de la ciudad y en otros puntos de Huancayo. Durante todos los meses del año se realizan distintas actividades en las principales calles; desfiles, procesiones, movilizaciones sociales, protestas sindicales, celebraciones, entre otros, entonces las calles y avenidas se congestionan, porque Huancayo cuenta con el segundo parque automotor a nivel nacional.

Existe una gran cantidad de pollerías en la calle Real y calles aledañas, causando una gran contaminación atmosférica y sonora; Huancayo como una ciudad en expansión y moderna no cuenta con una planta de tratamiento de residuos sólidos ni líquidos, sino con botaderos ocasionales lo que origina una gran incomodidad para la población y aún para pueblos circundantes, pues los botaderos se encuentran en distintos lugares (Pilcomayo, Paccha y Sicaya)

En relación con la seguridad, la ciudad se ha convertido en una localidad violenta y con altos índices de delincuencia, sobre todo por la presencia de bares, discotecas y centros nocturnos; que se trata de controlar a través de una seguridad particular y las municipalidades brindan seguridad mediante el serenazgo público.

Respecto al uso de los espacios públicos también deviene en una situación problemática, no existen áreas verdes ni de esparcimiento dentro de la ciudad, las población tiene el hábito de arrojar sus residuos sólidos y líquidos en las calles, y en los ríos, hacen sus necesidades fisiológicas en calles y parques, en 
épocas de lluvia. En los meses de diciembre a marzo, son frecuentes las inundaciones en muchas calles de la ciudad causando incomodidad a los transeúntes y a la población, el desorden que impera en los principales mercados de la ciudad es notorio con el comercio ambulatorio que invade aceras y pistas, los comerciantes hacen casi lo mismo, siendo el perjudicado el transeúnte y el consumidor.

A través de este trabajo de investigación nos hemos propuesto: investigar el uso de los espacios públicos en la ciudad de Huancayo. Como objetivos específicos nos trazamos los siguientes: determinar los aspectos sociales y culturales que concurren en la utilización de los espacios públicos en la ciudad de Huancayo y diseñar una propuesta social para el uso adecuado de los espacios públicos en la ciudad de Huancayo. Las hipótesis que hemos planteado son: el uso de los espacios públicos en la ciudad de Huancayo es inadecuado; no son espacios donde existan el respeto, la comunicación, ni la negociación, al contrario son espacios donde converge la violencia, la intolerancia y la irresponsabilidad. El uso de los espacios públicos es una situación cultural donde se aprecia el comportamiento y los hábitos que ha desarrollado la población y donde la responsabilidad es compartida por las personas y por las autoridades de la ciudad. Este uso inadecuado tiene que cambiar con la participación de la población, se tiene que crear una conciencia ciudadana y cívica, el cambio debe de ser a través de una propuesta social y cultural. Para lo cual realizamos primeramente una descripción de los usos del espacio público que dan las personas en Huancayo y luego analizamos e interpretamos estos usos.

\section{MATERIALES Y MÉTODOS}

El método utilizado en la presente investigación fue el hermenéutico, entender e interpretar los comportamientos sociales cotidianos desde la teoría social, la cultura y el estudio de las subjetividades. Para efectos de la descripción hemos realizado una observación de la vida cotidiana en estos espacios, en los mercados desde muy temprano en la mañana, en los momentos donde comienza la atención al público y el movimiento comercial. En los otros lugares hemos tomado como punto de referencia cualquier día de la semana ya que en estas zonas no existe un gran movimiento como en los mercados. Entrevistas no estructuradas con comerciantes de la zona para que nos relaten algunas características especiales de estos espacios y su mirada respecto al problema tratado en la investigación.

\section{RESULTADOS}

El mercado modelo, Es un interesante espacio para realizar estudios sociales porque confluyen gran cantidad de personas de distintas partes de la ciudad de Huancayo, de los distritos y comunidades cercanas y lejanas, hasta de la región de Huancavelica y Ayacucho (muy cerca existen agencias de transporte que se trasladan a estas regiones). Se puede distinguir a las mujeres con su vestimenta tradicional, faldín, lliclla y sombreros, lo que permite identificarlas de que parte del valle son 0 si son de otras regiones, muy pocos varones andinos mantienen la ropa tradicional, un elemento que aún se observa son los sombreros, pero la mayoría utiliza la ropa moderna, zapatillas, jeans, camisa, chompas, casacas, jockeys, se puede apreciar el uso de colores alegres en la vestimenta, rosado, celeste, verde claro, rojo, amarillo, etc., otro elemento multicultural y atractivo es la comida, su presentación es vistosa y colorida, con un fuerte aroma, además que se puede encontrar de distintos precios de acuerdo al prestigio que el platillo goza en las mesas populares y en los restaurantes, la mayor parte es de la región, son considerados como parte de la identidad del huancaíno, pero otros han sido traídos y adaptados de otras regiones del país.

Muchos migrantes han establecido sus negocios, de diferentes rubros en el mercado. En este espacio se congregan personas de distinta condición socio económica y racial. Se nota la concurrencia de pobladores de distritos y comunidades cercanas, especialmente mujeres que traen al mercado productos agrícolas, papa, maíz, arvejas, habas, granos, etc., para venderlos al menudeo, directamente al consumidor 0 a intermediarios y así complementar su economía familiar, también traen animales menores en "pie" (vivos); cuyes, conejos, gallinas, palomas, etc., o carcasas (animales muertos) los cuales son vendidos a los terceros, "jalachos", que esperan en las cercanías de los paraderos (Av. Ferrocarril) y al ver a las señoras que bajan de los autobuses con 
sus costales, se acercan a ellas y les compran sus animales, a bajo precio por supuesto.

Este mercado junto con el Mayorista o Raez Patiño, son los más importantes de la región y de la zona central del país, por su especial ubicación se abastece de todos los productos agrícolas y pecuarios del valle, de las otras provincias de la región Junín, también recibe los productos de la selva central, principalmente las frutas, y su cercanía a la capital le permite abastecerse de otros productos agrícolas, aves (pollos y sus derivados) y frutas, pero principalmente de abarrotes $\mathrm{e}$ insumos manufacturados.

El mercado Modelo está ubicado en el cercado de Huancayo, entre las siguientes calles; Jr. Huánuco por el sur, Cajamarca por el norte, Mantaro por el oeste y la avenida Ferrocarril por el este, el movimiento comercial empieza a las 6:30 am hasta las 5: 00 pm. aproximadamente. En este mercado se comercializa una gran cantidad de artículos de pan llevar, además de ropa y zapatos (en el segundo piso de este local), en su interior existe un relativo orden ya que la compra y venta esta dividida por sectores especializados, existe una zona de venta de carnes; con sus respectivas subdivisiones: de aves, res, pescados y mariscos, de carnero, menudencias y embutidos, sección de abarrotes, de condimentos, de molidos, de verduras, de panes, de comida, de jugos y gelatinas, de quesos, de huevos, de plásticos, entre otros. En los pasillos se aprecia personas que en forma ambulatoria expenden algunos artículos como plásticos, especies y otros, sin embargo, también existe una zona donde se venden animales menores en pie (aves de corral, conejos, cuyes, patos y otros) y mascotas como gatos, ratoncitos blancos y aves (periquitos australianos), el inconveniente con esta zona es que está al interior del mercado y los excrementos, plumas, pelos y otros desechos de estos animales se encuentran casi en contacto, a unos metros con la sección donde se comercializa la carne y el pescado. Muchas veces ha existido la intención de las autoridades municipales de reubicar a estos comerciantes en otros sitios, pero sin ningún éxito. Este local cuenta con servicios higiénicos que puedan utilizar los comerciantes y cualquier otra persona, siempre y cuando pague un determinado monto por el uso.
El verdadero problema se puede apreciar en los alrededores de este mercado, en las calles adyacentes. Éstas se encuentran prácticamente invadidas por los ambulantes que ofrecen distintas mercancías; pero, lo que complica la situación es que los productos se venden y en el suelo, en medio del polvo o del barro, no existe las condiciones mínimas de higiene, esto se aprecia sobre todo en la avenida Ferrocarril, donde las personas que vienen de algunas comunidades o distritos del entorno de la ciudad, a vender sus productos al por menor, que han cosechado de sus chacras, tienden un plástico o una manta en el suelo y encima ponen sus productos, y de esa manera se comercializa, la gente compra estos productos porque son más baratos en comparación a los que se expenden al interior del mercado, además en esa avenida la gente se ha acostumbrado a hacer sus necesidades fisiológicas, en época de lluvia esta parte se convierte en un lodazal y no es extraño ver gente alcohólica, vagabunda, enfermos mentales 0 del mal vivir, durmiendo en estas calles, incluso hay perros callejeros paseando los que también realizan sus necesidades en la calle.

Otro problema lo constituye el transporte que circula por estas calles, ómnibuses, camionetas rurales, taxis, mototaxis, si uno se traslada de sur a norte, desde el jirón Tarapacá hasta la calle Ica y si uno se moviliza de norte a sur desde la calle Ica hasta el jirón Tarapacá, se aprecia una congestión automotriz, los carros paran donde deseen, en medio de la pista 0 en medio de la cuadra, se demoran lo que quieren, esperando que suban pasajeros y que se llene su movilidad, muchas no respetan las señales de tránsito ni a la policía que se encuentra encargada de la circulación de esta zona, no hay respeto a la autoridad, esta situación se agrava cuando los carretilleros y tricicleros se desplazan por éstas calles.

Son por estos espacios que las personas se tienen que desplazarse para realizar sus compras, las aceras e incluso pistas invadidas por ambulantes y por tiendas que han sacado a la vereda y a las pistas su mercaderías (para llamar la atención), se aprecian cajas y sacos de mercaderías, los restaurantes han puesto sus rótulos, en la práctica no existen veredas para los peatones, entonces los transeúntes tienen que bajar a la pista para movilizarse, pero en la pista también se encuentran los ambulantes, entonces, ¿por dónde pa- 
san los vehículos y las personas?, debemos de tener en cuenta que estamos hablando de un mercado, donde transitan vehículos pesados de carga y la afluencia de personas es alta.

El gobierno local a través de la policía municipal intenta poner orden, desalojando a los ambulantes y decomisando sus mercaderías, pero este tipo de control solo se ejerce con el débil e indefenso, con el ambulante que probablemente es un vendedor ocasional sobre todo si es mujer o un campesina que se encuentra vendiendo un poco de sus cosechas, no existe sanción contra los comercios formalmente establecidos, que sacan a exhibir sus mercaderías a la vereda e incluso a las pistas, o contra los camiones que se estacionan donde les parece y que realizan tareas de descarga en las horas de gran afluencia de público ,dificultando el tránsito de peatones y vehículos.

El mercado genera diariamente grandes cantidades de residuos sólidos, las verduras, frutas y otras mercaderías perecibles que no se venden y por lo tanto se descomponen son arrojados a la vía pública, al transitar por éstas, nos encontramos con montículos de residuos, en plena descomposición, en las madrugadas, de 5 a 6 a.m. se puede observar en una de las puertas del mercado (Huánuco y Mantaro), un gran montículo de residuos sólidos que son recolectados por el servicio de limpieza pública de Huancayo, pero en el transcurso del día los comerciantes y el público arrojan distintos tipos de residuos sólidos en las esquinas y en cualquier lado de las calles.

Como todo mercado también se puede apreciar la venta de comida en establecimientos formales (dentro y fuera del mercado) y en forma ambulatoria. En Ios establecimientos formales se nota una relativa limpieza, porque existe una supervisión sanitaria por parte del Consejo Municipal de Huancayo, aunque los vectores, como las moscas y la contaminación del aire por los aspectos descritos, ocasionan problemas en la calidad ambiental de sus establecimientos y servicios. También existen establecimientos informales que venden comida, en carretillas 0 en la mismas aceras, que por sus bajos precios atraen a personas de pocos recursos económicos, la limpieza de sus utensilios se hace en un 0 dos baldes, que contienen agua que no se cambia, algo similar sucede con los que venden jugos, extractos, gelatinas, refrescos, chupetes en forma ambulatoria. Los residuos que generan este tipo de negocios también son arrojados a la vía pública.

El edificio del mercado cierra sus puertas a las 5 de la tarde, pero en sus contornos comienza otro estilo de vida, la avenida de Ferrocarril se llena de ambulantes que venden ropa y zapatos usados a cómodos precios, se vende comida, la cena, pescado frito, salchipapas, pollo broster, entre otros, hay bastante movimiento comercial y de transporte, se reproduce las condiciones de desorden y falta de higiene del turno diurno.

A una cuadra del mercado modelo, en la esquina de la avenida Ferrocarril y el Jr. Tarapacá, se puede apreciar un centro de beneficio de aves, grandes camiones llegan en forma ínter diaria trayendo los pollos de la costa, el olor a excremento se siente a más de 100 metros a la redonda, luego de haber beneficiado a las aves, baldean el local, la sangre y las plumas salen hasta la calle, juntándose gran cantidad de moscas en esta parte de la ciudad, en épocas de navidad, año nuevo y otras fechas festivas la situación se agrava por el mayor número de aves que se comercializa.

\section{DISCUSIÓN}

Esta es la parte interpretativa de la investigación hemos descrito el problema y la situación del uso de los espacios públicos de la ciudad de Huancayo, pero debemos analizar a qué factores obedecen estos hechos y cómo cambiarlos.

Muchas personas piensan que la educación es el eje principal por la cual una sociedad se va a desarrollar, no están lejos de la verdad, pero la antropología considera el proceso de socialización mediante el cual el hombre aprende sus roles, hábitos y a comportarse dentro de su grupo social, habría que examinar si en nuestro medio se socializa al individuo para respetar las normas de convivencia. Según el sociólogo Max Weber, los países de la parte central, norte y occidental de Europa lograron un desarrollo por su respeto a las normas. 
En las escuelas, en los colegios, en las universidades, en los programas peruanos que transmiten los medios de comunicación, en la familia y en otras instituciones que socializan al individuo se valoriza más al que trasgrede las normas sociales que a aquél las respeta, el líder en los colegios es el que practica la anticultura, el que incumple con sus deberes, no cumple con sus trabajos, el "vivo", esto se reproduce en la universidades, en los trabajos, las autoridades peruanas son un buen ejemplo, como lo refiere Juan Carlos Ubilluz, los más grandes pendejos en la historia del Perú fueron; el ex presidente Alberto Fujimori y su asesor Vladimiro Montesinos.

G. Portocarrero (2004) indica que en la sociedad peruana la ley pública no tiene prestigio, la "desviación" deja de ser excepcional para convertirse en un comportamiento institucionalizado, entonces, la corrupción y el abuso con los débiles se convierten en hechos "normales", desarrollándose así una tolerancia a la trasgresión que socava el orden moral, y la sociedad le da la espalda a los valores y normas que estamos obligados a acatar. Además la trasgresión a la norma acarrea un goce en el trasgresor sobre todo cuando no es descubierto y su conducta queda impune, reitera que los gobernantes desde la época colonial eran los ejemplos de irrespeto a las normas, estos comportamientos fueron copiados rápidamente por otros sectores sociales, y en la época republicana de mitad del siglo XX, en el proceso de migración andina a la costa y a la capital, se da un proceso de acriollamiento y avivamiento de los migrantes.

En el espacio de la presente investigación del uso de los espacio públicos se puede observar cotidianas formas de trasgresión a la norma social, sobre todo en el transporte público, la persona que respeta las normas de tránsito es vista como tonta, no es un pendejo, su posibilidad de goce esta recortada por un sistema de valores que funciona en nuestra sociedad y que se resume en la siguiente frase popular; el tonto vive de su trabajo y el vivo vive del tonto, al final si un policía de tránsito de captura al cometer una infracción existe la gran posibilidad de sobornarlo y si nadie se da cuenta pasas como una gran "vivo" e incluso lo puedes contar como un anécdota en otra ocasión.

\section{CONCLUSIONES}

- Existe un inadecuado uso de los espacios públicos en la ciudad de Huancayo donde se presenta la violencia, la intolerancia y la irresponsabilidad debido a una mala socialización del individuo, se han impuestos modelos relacionados con la criollada y la pendejada antes que modelos cívicos de respeto y de derechos - obligaciones.

- No creemos que la pobreza económica justifique estos hechos. La responsabilidad de esta situación es compartida entre autoridades - ciudadanos, transportistas - usuarios, comerciantes - clientes.

- El cambio no pasa por propuestas técnicas, leyes más rígidas, campañas temporales o cambio de autoridades, sino por la elaboración e institucionalización de políticas culturales donde se valorice el respeto a las normas y a la persona.

\section{LITERATURA CITADA}

Amat, C. 2006. El Perú nuestro de cada día. Edit. Universidad del Pacífico. Lima.

Arguedas, J. 1982. Dos estudios sobre Huancayo. Edit. UNCP. Huancayo.

Augé, M. 2006. Los no lugares. Espacios del anonimato. Una antropología de la sobre modernidad. Edit. GEDISA .Barcelona.

Beck, U. 1998. La sociedad del riesgo. Hacia una nueva modernidad; Edit. Paídos. Buenos Aires.

Benhabib, S. 2006. El ser y el otro en la ética contemporánea. Feminismo, comunitarismo y posmodernismo. Edit. GEDISA Barcelona.

Bourdeu, P. 1996. La dominación masculina. Edit. Anagrama. España.

Conrad, P. 2006 Antropología cultural. Edit. Mc Graw Hill. España. 
Degregori, C. 1986. Conquistadores de un nuevo mundo. De invasores a ciudadanos en San Martín de Porras. Edit. IEP. Lima.

De Soto, H. 1987. El otro sendero. Edit. Instituto libertad y democracia. Lima.

Figueroa, A. 1996. Exclusión social y desigualdad en el Perú. Edit. PNUD Lima.
Giddens, A. 2004. Sociología. Edit. Alianza. Madrid.

Golte, J. y Adams, N. Los caballos de Troya de los invasores, estrategias campesinas en la conquista de la Gran Lima. Edit. IEP. Lima. 